\title{
Commentary: Synthetic Strigolactone analogues reveal anti-cancer activities on hepatocellular carcinoma cells
}

\author{
Razvi SS ${ }^{1,2,3 *}$, Hasan MN1, Moselhy SS ${ }^{1,4}$, Kumosani TA ${ }^{1,5}$, Al-Malki AL ${ }^{1,6}$, Kumosani AT ${ }^{7}$ and Asami T T,8 $^{1,8}$ \\ ${ }^{1}$ Department of Biochemistry, Faculty of Science, King Abdulaziz University, Jeddah, Saudi Arabia \\ ${ }^{2}$ M.S Research Foundation, Hyderabad, India \\ ${ }^{3}$ Department of Genetics, Vasavi Medical and Research Center, Hyderabad, India \\ ${ }^{4}$ Bioactive Natural Products Research Group, King Abdulaziz University, Jeddah, Saudi and Biochemistry Department, Faculty of Science, Ain Shams University, \\ Cairo, Egypt \\ ${ }^{5}$ Experimental Biochemistry Unit, King Fahd Medical Research Center and Production of Bioproducts for Industrial Applications Research Group, King Abdulaziz \\ University, Saudi Arabia \\ ${ }^{6}$ Experimental Biochemistry Unit, King Fahd Medical Research Center and Bioactive natural products Research Group, King Abdulaziz University, Saudi Arabia \\ ${ }^{7}$ Department of Pathology and Laboratory Medicine, Blood Bank Transfusion Medicine, King Abdulaziz Medical city, Jeddah, National Guards, Jeddah, 21423, \\ Saudi Arabia \\ ${ }^{8}$ Graduate School of Agricultural and Life Sciences, University of Tokyo, Bunkyo, Tokyo 113-8657, Japan
}

We recently published a research article [1] on the anti-proliferative properties of the Novel synthetic Strigolactone (SLs) analogues in Hepatocellular carcinoma (HCC). In this article, we reported the decrease in the percentage of cell viability after exposure of HepG2 cells to different SLs and the pro-apoptotic effect through flow cytometry. We also evaluated the anti-invasive potential of the SLs in the suppression of cell migration and healing the wound after exposure to varying doses of SLs. In this commentary, we have tried to put forward the key findings in the article and the possible future research directions.

To the best of our knowledge, this is the first ever anti-proliferative and anti-invasive study conducted on HepG2 cells with these new derivatives. We hypothesized that the SLs could inhibit the growth of HepG2 cells as evidenced by the previous studies on breast and prostate cancer $[2,3]$. The rationale of this study was to observe the anti-proliferative effect of the newly synthesized SLs at varying concentrations. Initially, we investigated the anti-proliferative effect of SLs in a dose-dependent manner through WST-1 assays. It is wellknown that apoptosis is differentiated from other forms of cell death by the rate of sequential decrease in the proliferating cells with the increase in the dose [4]. Hence, to confirm the possibility of a pro-apoptotic effect, we further studied the inhibitory impact of SLs in a timedependent manner by retaining a constant dose of $80 \mu \mathrm{M}$ throughout this assay; in contrast to apoptosis, in other forms of cell death, there is a sudden decrease in the cell viability. However, we observed that there was a time-dependent decrease in the cell viability when the cells were treated with different SLs. Furthermore, we were curious to examine the effect of the SLs on additional cell lines like PC3 (prostate cancer) and Jurkat (T-cell lymphoblastic leukemia) to build upon an earlier study by our group which examined pro-apoptotic effect of acyl spermidine derivatives on Jurkat cells [5]. Surprisingly, we found that SLs-TIT3 and TIT7 also showed an antiproliferative impact against PC3 and Jurkat.

Additionally, it is also crucial to corroborate the study by analysing the effect of the drug on healthy cells (non-cancerous). Therefore, we tested the selected SLs on baby hamster kidney (BHK) cells and found that there was minimal toxicity at the highest concentration with a significant decrease in the cells, which is not uncommon. Honestly, no anti-cancerous drug has been developed to date which doesn't show off-target toxicities besides being highly efficacious in treatment [6]. Nevertheless, the researchers and clinicians cautiously weigh the 'pros' and 'cons' of a pro-drug molecule before arriving at a definite conclusion for its incorporation in existing treatment regimens [7].

It is worth mentioning that apoptosis is favoured over necrosis in reducing tumorigenicity; necrosis is "unprogrammed" form of cell death characterized by the inflammation of cell membrane, membrane disruption and release of intracellular contents to the external milieu which is quite detrimental [8]. On the contrary, apoptosis is a programmed cell death process which activates an irreversible cascade of reactions through successive activation of different caspases and protein complexes without any membrane disruption and deleterious effects [9].

Therefore, we next examined the effect of the selected SLs on the tumour cells through Annexin V/7AAD staining by using flow cytometry. Phosphatidylserine (PS) is present in the inner leaflet of the plasma membrane which is exposed to the exterior when the cell membrane is compromised [10]. Annexin V is a specific Ca+2 dependent protein with high affinity towards PS and is tagged with a fluorescent probe detected by flow cytometry. We used a mixture of Annexin V-PE and 7AAD (7-amino actinomycin D) which is a nuclear stain. The results give a typical image of four quadrants; live cells are shown in lower-left, early apoptotic cells in lower-right followed by lateapoptotic cells in upper-right and dead cells in upper-left quadrants. We measured the pro-apoptotic effect by annexin staining in both dose-

${ }^{\star}$ Correspondence to: Syed Shoeb Razvi, Department of Biochemistry, King Abdulaziz University, Saudi Arabia, E-mail: shoebiqbal@hotmail.com

Key words: strigolactone analogues, HepG2, apoptosis, wound healing, HCC

Received: August 06, 2018; Accepted: August 20, 2018; Published: August 27, 2018 
and time-dependent manner to substantiate our previous findings. Consequently, it was appealing to note that most cells were found in the lower-right quadrant which increased significantly in a dose-dependent manner.

Finally, we also investigated the potential of the SLs in suppressing the migration of cancer cells through a wound healing assay. It is well documented in the literature that the in vitro cell migration assays give us a basic idea of the potential of a drug molecule to suppress the invasiveness of the tumours [11]. Hence, we made a wound from scratch on the confluent monolayer of HepG2 cells to examine the potential of SLs in inhibiting the wound healing. Unanticipatedly, much to our exhilaration, the selected compounds TIT3 and TIT7 both demonstrated a significant positive inhibitory effect compared to the control. The SLs effectively suppressed the healing of wound by inhibiting the cell migration into the scratched area. Hence, we strongly recommend further investigation of the SLs through molecular and genetic expression studies to deduce an attractive therapeutic target which can be aimed in designing an effective therapeutic regimen. However, we also suggest animal studies with HCC models to confirm the in vitro results, as there is a considerable difference between the passive and dynamic settings of in vitro and in vivo conditions respectively.

\section{Conflicts of interest}

The authors declare that no financial conflict of interest exists for the present study.

\section{References}

1. Hasan MN (2018) Synthetic strigolactone analogues reveal anti-cancer activities on hepatocellular carcinoma cells. Bioorg Med Chem Lett 28.

2. Pollock CB, McDonough S, Wang VS, Lee H, Ringer L, et al. (2014) Strigolactone analogues induce apoptosis through activation of $\mathrm{p} 38$ and the stress response pathway in cancer cell lines and in conditionally reprogrammed primary prostate cancer cells. Oncotarget 5: 1683-1698. [Crossref]

3. Pollock CB, Koltai H, Kapulnik Y, Prandi C, Yarden RI (2012) Strigolactones: A novel class of phytohormones that inhibit the growth and survival of breast cancer cells and breast cancer stem-like enriched mammosphere cells. Breast Cancer Res. Treat 134 1041-1055. [Crossref]

4. Potten CS, Wilson JW, Booth C (1997) Regulation and significance of apoptosis in the stem cells of the gastrointestinal epithelium. Stem Cells 15: 82-93. [Crossref]

5. Razvi SS (2017) Synthesis, screening and pro-apoptotic activity of novel acyl spermidine derivatives on human cancer cell lines. Biomed. Pharmacother 93: 190201

6. Ventola CL (2017) Cancer immunotherapy, part 3: Challenges and future trends. $P T$ 42: 514-521. [Crossref]

7. Rodrigues DG (2007) Clinical research and drug development in Latin America: weighing the pros and cons, talking about the future. J Investig Med 55: 223-229. [Crossref]

8. Lee SY, Ju MK, Jeon HM, Jeong EK, Lee YJ, et al. (2018) Regulation of tumor progression by programmed necrosis. Oxid Med Cell Longev 2018: 3537471. [Crossref]

9. Zornig M, Hueber A, Baum W, Evan G (2001) Apoptosis regulators and their role in tumorigenesis. Biochim Biophys Acta 1551: F1-37. [Crossref]

10. Watson H (2015) Biological membranes. Essays Biochem 59: 43-69. [Crossref]

11. Justus CR, Leffler N, Ruiz-Echevarria M, Yang LV (2014) In vitro cell migration and invasion assays. $J$ Vis Exp. [Crossref]

Copyright: (C2018 Razvi SS. This is an open-access article distributed under the terms of the Creative Commons Attribution License, which permits unrestricted use, distribution, and reproduction in any medium, provided the original author and source are credited. 